\title{
AWireless Local Area Network Soft Cell Phone (SCP) System with Multimedia and Data Services
}

\author{
Olakanmi O.Oladayo \\ Electrical and Electronic Engineering, Technology Drive, Office 6, New Faculty of Engineering Building. \\ University of Ibadan, Ibadan Nigeria \\ Olakanmi.oladayo@ui.edu.ng
}

\begin{abstract}
Most organizations concurrently maintain private Automatic Branch Exchange (PABX) and Local Area Network (LAN) for information interchange within their organization. This is obviously a waste of resources and avoidable duplication of communication systems. The existing LAN can be used as a communication backbone for the in house telephone operations with no extra cost and resources. In view of this, a portable and a platform independent Software-Based Cell Phone (SCP) was proposed for the existing LANs infrastructure in the organizations.

The SPC is a telephony application with a user friendly interface which is capable of handling voice, video and text messages without compromising the Quality of Service (QoS) of the existing LAN.
\end{abstract}

Index Terms-Telephone, LAN, Voice, Internet Protocol, VoIP.

\section{INTRODUCTION}

Good communication services and universal access are necessary for a higher standard of living and economic growth [1]. The need to communicate with many people over a long distance ranges brought about the invention of telephone exchange. It was initially based on electronic Circuit Switched Networks (CSNs) allocating a dedicated circuit from end to end. Afterwards, another form of electronic communication in the form of data was also invented. The two forms have continuously experienced improvement ever since. These improvements have led to the fall in price of communication devices especially those for data. As a result, the growth of Local Area Network (LAN) usage in homes and organizations has increased. LANs are typically used in many homes and organizations for file sharing and for synchronizing activities during the execution of team works. LAN connections vary from wired connections to wireless connections with varying speed of connection and available bandwidth.

In the developing economy, financial resources are limited and the cost of communication is quite high. Hence, other cheaper means of communication are usually explored. One of the recent advancements is Voice over Internet Protocol (VoIP) applications. It is the convergence of voice and data communication systems. Better still, Voice over IP can be described as amethodology and group of technologies for the delivery of voice communications and multimedia sessions over Internet Protocol (IP) networks, such as the Internet. Other terms commonly associated with VoIP are IP telephony, Internet telephony, voice over broadband (VoBB), broadband telephony, IP communications, and broadband phone service.

The term Internet telephony specifically refers to the provisioning of communications services (voice, fax, SMS, voice-messaging) over the public Internet, rather than via the public switched telephone network (PSTN). The steps and principles involved in originating VoIP telephone calls are similar to traditional digital telephony, and involve signaling, channel setup, digitization of the analog voice signals, and encoding. Instead of being transmitted over a circuit-switched network, however, it employs packet switched network. That is the digital information is packetized and transmission occurs as Internet Protocol (IP) packets over a packet-switched network.

Early providers of voice over IP services offered business models and technical solutions that mirrored the architecture of the legacy telephone network. When such technologies are deployed over a LAN, then it is called LAN telephony [2]. This has brought about the design and implementation of various forms of Voice over Internet Protocol (VoIP) applications. An example of this is the proposed SCP system. The remaining part of this paper is arranged as follows: section 2 contains reviews of some related VoIP telephone systems. The design methodology and working principle of the SPC system are explained in section 3 . Section 4 contains the test results and conclusion.

\section{RELATED WORKS}

The development of communication system began with Public Switched Telephone Network (PSTN) and evolved continuously to wireless and Internet Protocol (IP) technologies [3] The internet is the data counterpart of the PSTN [4]. The internet was not originally designed to carry audio. However, with recent development in internet technologies, Voice over Internet Protocol (VoIP) system was invented which enables human voice to be transmitted using IP.

The development of VoIP began with researchers at Massachusetts Institute of Technology (MIT) in the mid- 
1970s. It was first limited to the academic environment. Developments then continued with the introduction of networks with faster speed and larger bandwidth capacity. The deployment of Voice over Internet Protocol (VoIP) applications offer better advantages compared to the use of PABX systems. Some of the VoIP like Skype and Yahoo Video and Voice Chat' are available as off-the-shelve software applications. Meanwhile, a few of these are features limited while others are expensive with exorbitant monthly or annual subscriptions. Also, most of them are better adapted for usage over the internet.

VoIP systems employ session control and signaling protocols to control the signaling, set-up, and tear-down of calls. They transport audio streams over IP networks using special media delivery protocols that encode voice, audio, video with audio codecs and video codecs as Digital audio by streaming media. Various codecs exist that optimize the media stream based on application requirements and network bandwidth; some implementations rely on narrowband and compressed speech, while others support high fidelity stereo codecs. Some popular codecs include $\mu$-law and a-law versions of G.711, G.722 which is a high-fidelity codec marketed as HD Voice by Polycom, a popular open source voice codec known as iLBC, a codec that only uses $8 \mathrm{kbit} / \mathrm{s}$ each way called G.729, and many others. Voice over IP has been implemented in various ways using both proprietary protocols, as well as protocols based on open standards. Examples of the VoIP protocols are H.323, Media Gateway Control Protocol (MGCP), Session, Initiation Protocol (SIP), Real-time Transport Protocol (RTP), Session Description Protocol (SDP), InterAsterisk eXchange (IAX), Jingle XMPP VoIP extensions and Skype protocol. The H.323 protocol was one of the first VoIP protocols that found widespread implementation of long-distance traffic, as well as local area network services. However, since the development of newer, less complex protocols such as MGCP and SIP, H.323 deployments are increasingly limited to carrying existing long- haul network traffic. In particular, the Session Initiation Protocol (SIP) has gained widespread VoIP market penetration. These protocols can be used by special-purpose software, such as Jitsi, or integrated into a web page (web-based VoIP), like Google Talk.

A major development that started in 2004 was the introduction of mass-market VoIP services that utilize existing broadband Internet access, by which subscribers place and receive telephone calls in much the same manner as they would via the public switched telephone network (PSTN). Full-service VoIP phone companies provide inbound and outbound service with direct inbound dialing. Many offer unlimited domestic calling for a flat monthly subscription fee. This sometimes includes international calls to certain countries. Phone calls between subscribers of the same provider are usually free when flat-fee service is not available A VoIP phone is necessary to connect to a VoIP service provider. This can be implemented in several ways: Dedicated VoIP phones connect directly to the IP network using technologies such as wired Ethernet or wireless $\mathrm{Wi}-\mathrm{Fi}$. They are typically designed in the style of traditional digital business telephones.

As stated earlier, the first generation providers of voice over IP services offered business models and technical solutions that mirrored the architecture of the legacy telephone network. Second generation providers, such as Skype, built closed networks for private user bases, offering the benefit of free calls and convenience, while potentially charging for access to other communication networks, such as the PSTN. This has limited the freedom of users to mix-and-match thirdparty hardware and software. Skype has 663 million registered users as of the end of 2010. Skype has also become popular for its additional features, including file transfer, and video conferencing. Competitors include SIP and H.323-based services, such as Linphone and Google Voice. Unlike most other VoIP services, Skype is a hybrid peer-to-peer and client-server system. It makes use of background processing on computers running. Apart from the subscription cost of Skype, it does not protect users' right to privacy. United State intelligence agency, National Security Agency (NSA), monitors Skype users' activities such as content of emails, chats, uploads and downloads of all non US nationals through their surveillance program PRISM. This infiltration although requires FISA court authorization jeopardizes users' privacy [2] [5] [6] [7]. Meanwhile third generation providers, such as Google Talk have employed the concept of federated VoIP which is a departure from the architecture of the legacy networks. These solutions typically allow dynamic interconnection between users on any two domains on the Internet when a user wishes to place a call [2] [5] [6] [7].

\section{THE PROPOSED WLAN SPC SYSTEM}

The design of the SPC system involves both network protocols and application protocols integration. In the LAN soft phone system design, a computer system is essential. The operation of the system can be divided into three main parts, speech collection/playing, codec (coder/decoder) and socket communication parts. At the transmitting end, an analogue speech signal is collected from the physical environment (user) via a computer microphone (line-in). The speech collected has superimposed background noise of the environment. The collected analogue signal is then converted to a digital signal through pulse code modulation technique (PCM) by the computer system soundcard. Each sampled height is represented in 16 bits. The digitized signal is compressed to 8 bits using codec technique before being transmitted via controlled socket communication over a Local Area Network (LAN) to the receiver. The signals may experience some delays during transmission. On getting to the receiver's end, they are decompressed back to 16 bits and then converted to analogue voice signals by the receiver's system soundcard before being played out by a speaker (line-out). 
The SPC user interface provides user access to initialize and end calls. Protocols and standards are employed in signaling, compressing, decompressing and transmission processes.Fig. 1 shows the block diagram of the soft cell phone system.

\section{A. Network Capacity Estimation for SPC}

The capacity of LAN over which a soft cell phone system is to be deployed was estimated. This helped in determining the appropriate codec technique to use and an idea of the number of users the LAN can adequately support. In this work, the bandwidth capacity of particular WLAN setup was estimated. For the DWA525 Wireless N 150 Desktop Adapter used in this WLAN, the maximum bandwidth $B_{\max }$ it can support as indicated by the manufacturer is $150 \mathrm{Mbps}$. However, due to the shared nature of wireless network communication, bandwidth estimation is far more challenging. The bandwidth actually available for use $B_{\max (a v a i l a b l e)}$ is not usually more than 0.8 of the available maximum capacity. This is because fluctuation and interference cause variation in wireless capacity and available bandwidth. For this work, a factor of 0.6 for maximum available bandwidth was used, therefore,

$$
\begin{aligned}
& B_{\max }=150 \mathrm{mbps}, \text { and assumed available factor } \\
& =0.6 \\
& B_{\text {available }}=(\text { assumed available factor } \\
& \text { * 150) } \\
& B_{\text {available }}=(0.6 * 150)=90 \mathrm{mbps}
\end{aligned}
$$

FortheWLAN to adequately support multimedia and data services without flooding the network, the available bandwidth must be shared equally for the two services. The bandwidth capacity allocated for multimedia service $B_{\max (\text { multimedia) }}$ therefore is:

$B_{\max (\text { multimedia })}=0.5 * B_{\max (\text { available })}$

\section{B. SPC System Design and Implementation}

At this stage, a software application for the SPC system was designed and developed. The software uses Internet Protocol Version 4 (IPV4) to locate recipient and Multimedia Access Control (MAC) address to uniquely identify clients. The software development is in stages leading to the development of four interwoven modules showing in table 1.

1. Main module for signalling, control and ease of accessibility.

2. Messaging module for messaging functionalities.

3. Voice module for voice call.

4. Video module for video call.

\section{a) Main Module Development}

It consists of a group of forms that provides an interface for signalling and ease of accessibility. The most important part of this module is the home screen panel. The major forms/sections in the module are:

1. Client login and password edit panel: The client login panel provides authentication facilities to prevent unauthorized usage. This password edit panel allows a user to change or reset login password.

2. Home screen panel: It displays status information about the application. It has a section for selecting accessibility options. It performs numerous background signalling and control operations.

3. Phone settings: It provides links shortcuts to accessibility operations and provides room for selecting the type of encoding (A-law, u-law or none) to be used for calls.

4. Network settings: It provides facilities for creating and managing network handles and groups.

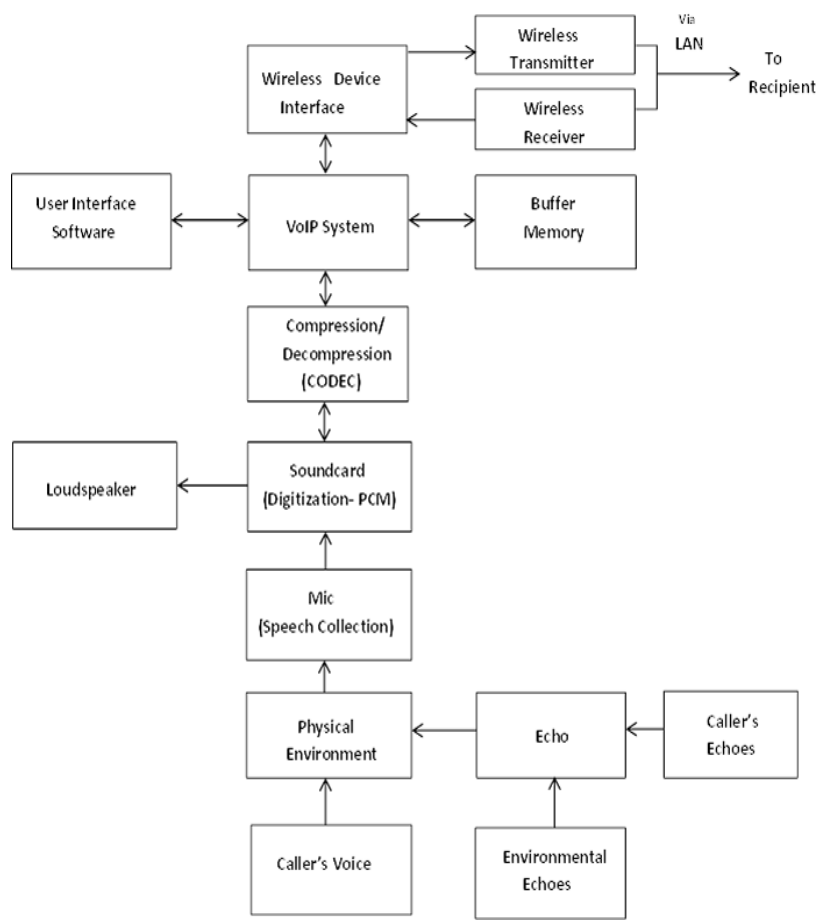

Figure 1: Block Diagram of Software-BasedLAN Telephony System

b) Messaging Module Development

It basically provides messaging facilities for composing and sending messages. Before a message (SMS) is sent, the online status of the recipient is confirmed. Fig.9 shows the flowchart for sending an SMS.

c) Voice Module Development

This is the main module for the actual voice communication. Before the module starts up, the recipient availability and readiness are confirmed at the task selection bar level or form stage. If conditions are satisfied, the voice module starts up and begins to dial the recipient. On acceptance of the call by the recipient, voice packet transmission begins and a timer to monitor the time duration is initialized. On the ending of call from either end, the timer is stopped and call details are saved in 'Voice Call Log' at both ends. If however, the 
recipient rejects the call, a 'user busy reply' is sent to the caller or if the call is left unanswered, a 'call not accepted reply' is sent to the caller after 30 seconds and then the call is ended and call details are saved at both ends to the 'Voice Call Log'. Fig.10 and Fig.11 shows the flowchart of the main processes involved in making a voice call at the caller's end and receiving voice call at the recipient's end respectively.

TABLE 1: Sections of Software Application.

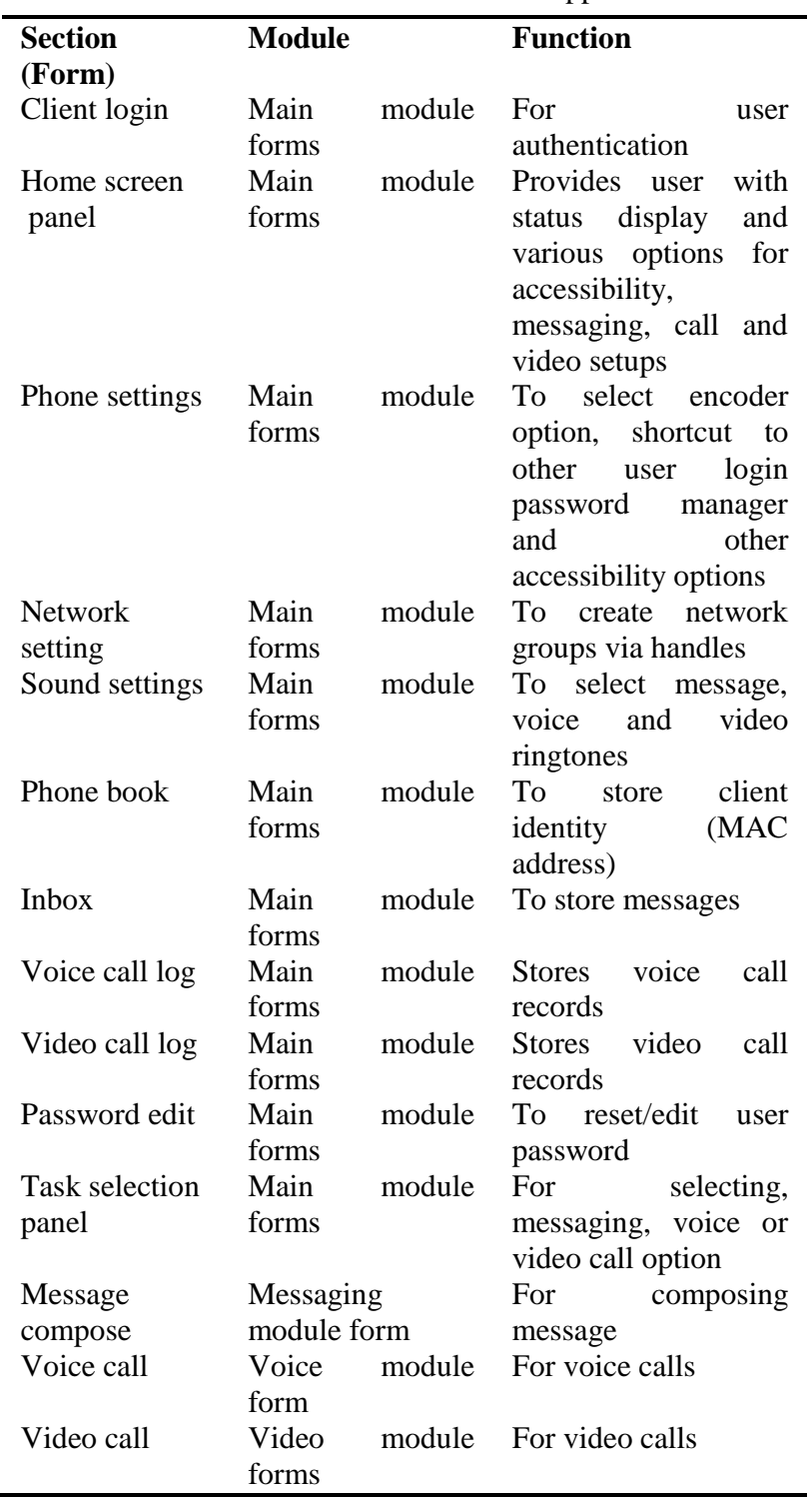

\section{VI.TESTS AND EVALUATION OF SPCSYSTEM}

\section{A. Testing of Main Module}

Fig. 2 shows the 'User Login Form' on running the SPC. From the 'User Login Form', the user can choose to reset the password or login by entering appropriate password. On entering the appropriate password, 'Home Screen Panel' is loaded as shown in Fig.3. At this stage, an attempt would be made by the SPC software to ping other online users in the group. The list of users online would be updated as users came online and went offline. The panel also displays link to accessibility options link 'Phone Settings', 'Sound Settings', 'Phone Book', 'Inbox', among others

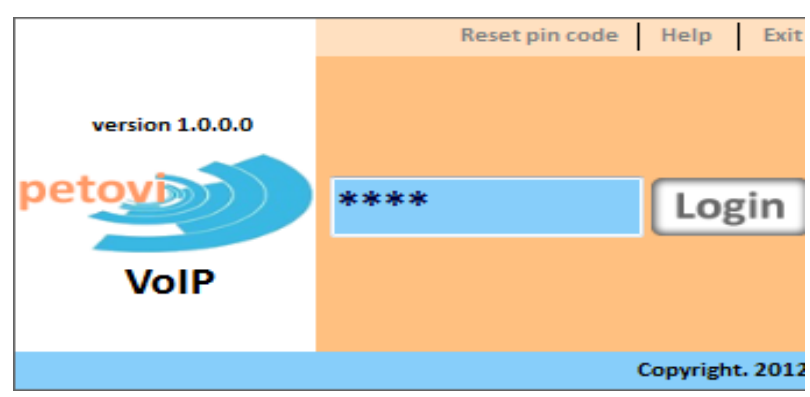

Figure 2: User Login Form

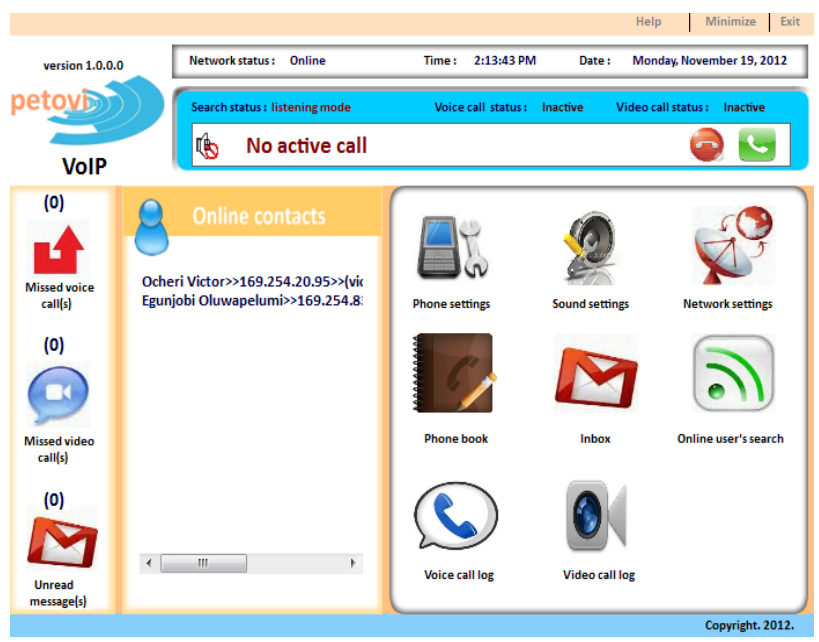

Figure 3: Home Screen Panel

To send a message or make a call (video a voice call), the 'Task Selection Bar' was loaded by double-clicking on the recipient from the online users list as shown Fig.4. From the bar, different forms are loaded depending on the option selected.

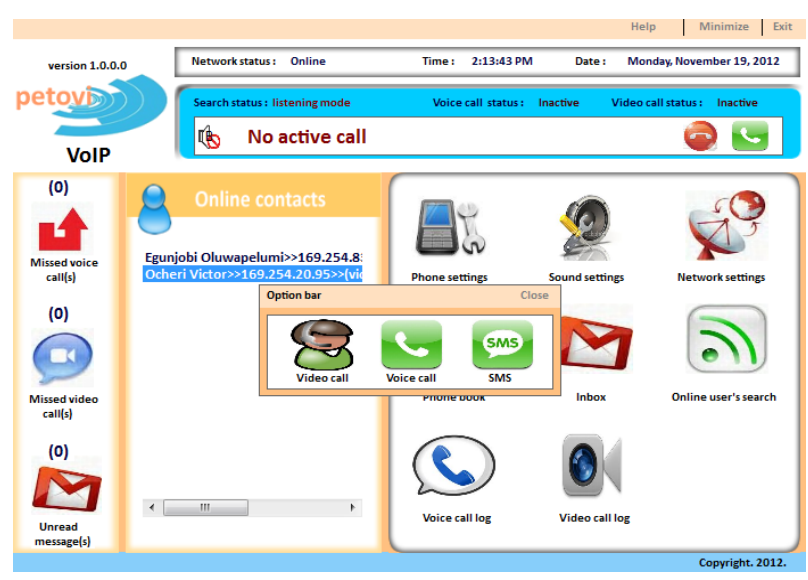

Figure 4: Home Screen Panel with Task Selection Bar

\section{B. Testing of Messaging Module}

On selecting 'SMS' option from the 'Task Selection Bar', the 'SMS Form' (messaging module) was loaded with the recipient address pre-loaded. The message was 
composed by typing in the compose field. When the 'Send' button was clicked after composing the message, an attempt to send the message was made. A response message was displayed on the screen indicating whether the message was sent or not. Fig.5 and Fig.6 are screenshots of the form when a message was being composed and after the message was successfully sent respectively.

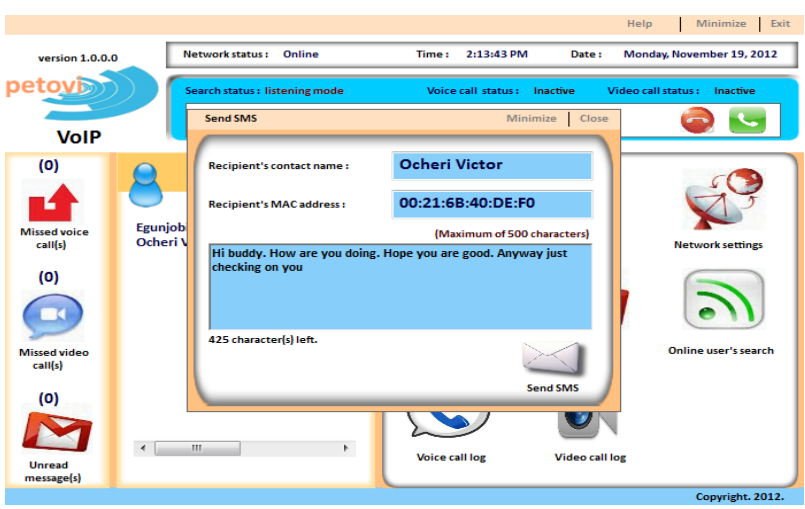

Figure 5: Message Compose Form

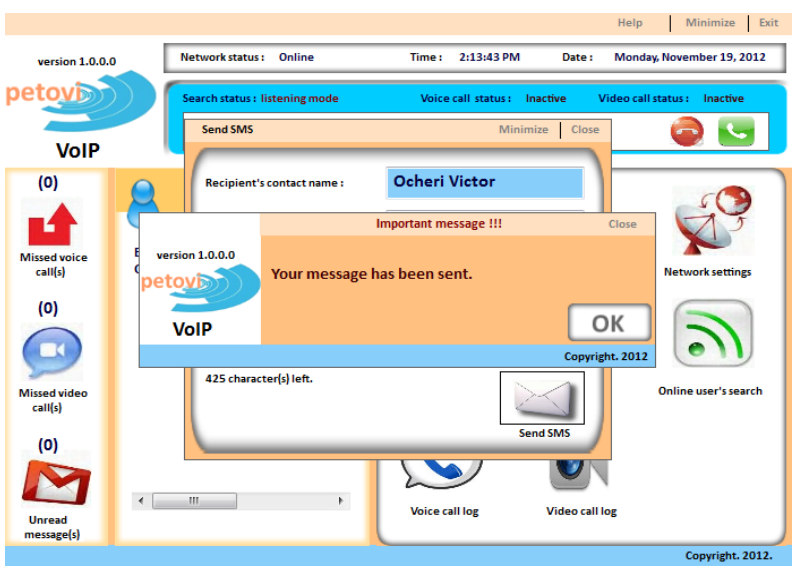

Figure 6: Message Compose Form with Delivery Report

\section{Testing of Voice Module}

On selecting 'Voice Call' option from the 'Task Selection Bar', the 'Voice Call Form' (voice module) was either loaded or not depending on whether the recipient was in a state to receive a call or not. The recipient is not in a state to receive a call if recipient suddenly goes offline or is already on another call. The caller was notified via a response message. If the recipient is in a state to receive a call, then the 'Voice Call Form' is loaded and an attempt to dial the recipient is made. The recipient was notified of an incoming call request as shown in Fig.7. If the recipient picks up the call, then the conversation starts as shown in Fig.8 and Fig.9 If the call is rejected, a response message showing that the call was rejected was displayed to the caller as shown in Fig.10. If the call is not picked after thirty (30) seconds then a reply indicating that the call was not picked was displayed to the caller. Fig.11 shows the screenshot of the form on ending a call after some period of conservation.

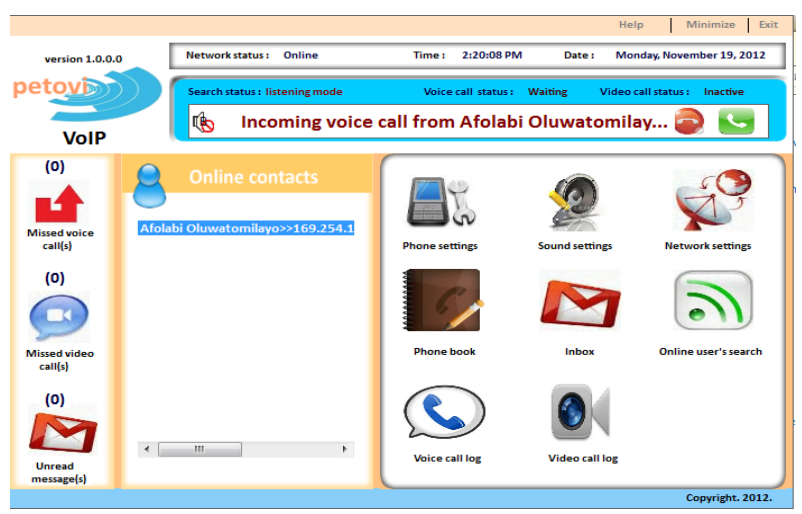

Figure 7: Voice Call in Progress (Caller's End)

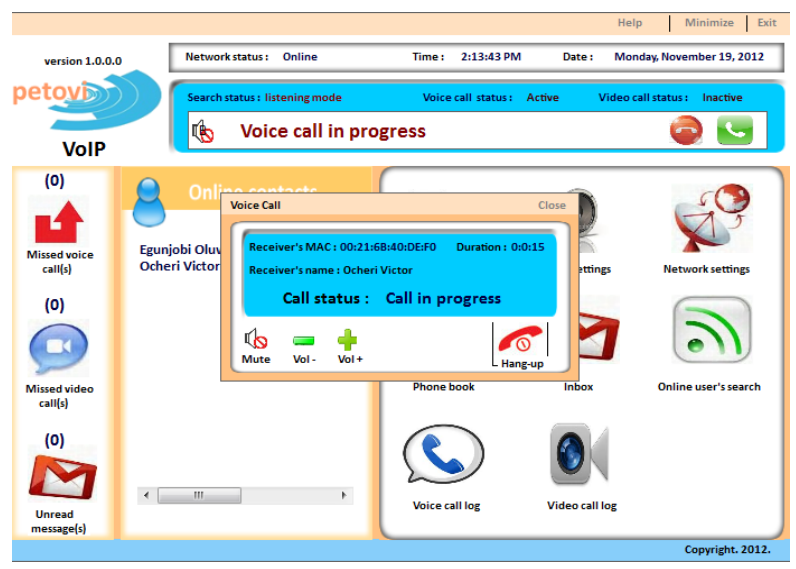

Figure 8: Incoming Voice Call Request

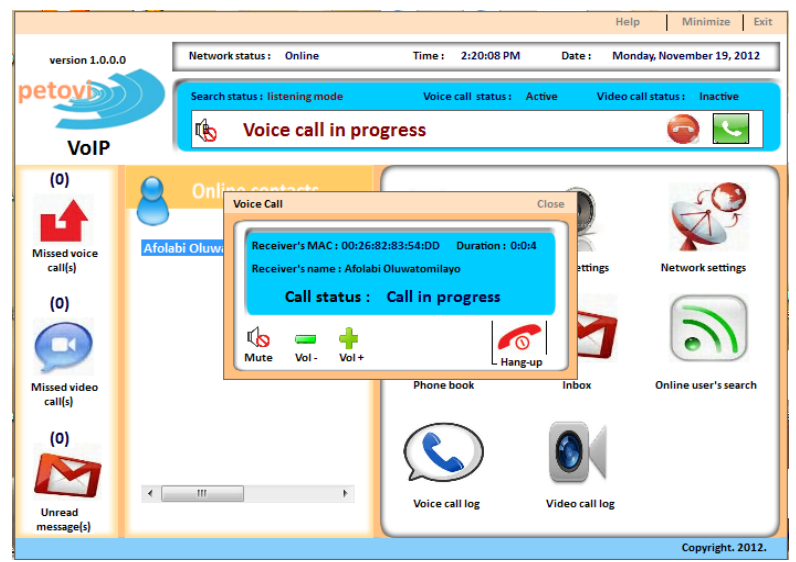

Figure 9: Voice Call in Progress (Receiver's End)

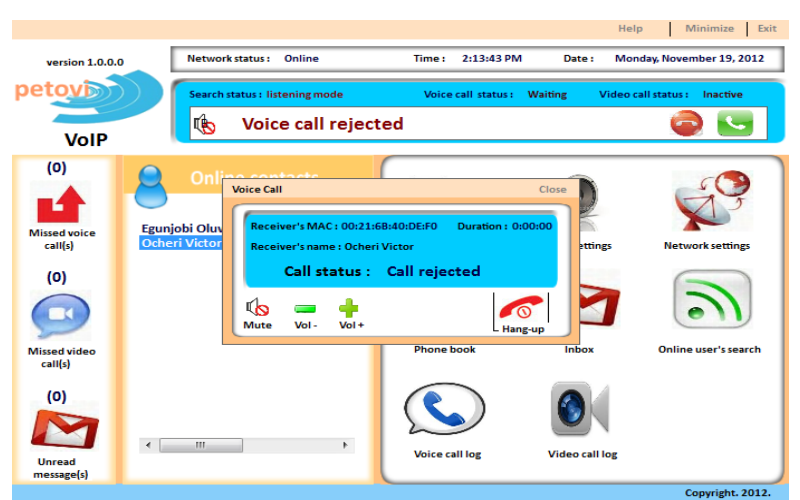

Figure 10: Voice Call Rejected by Receiver 


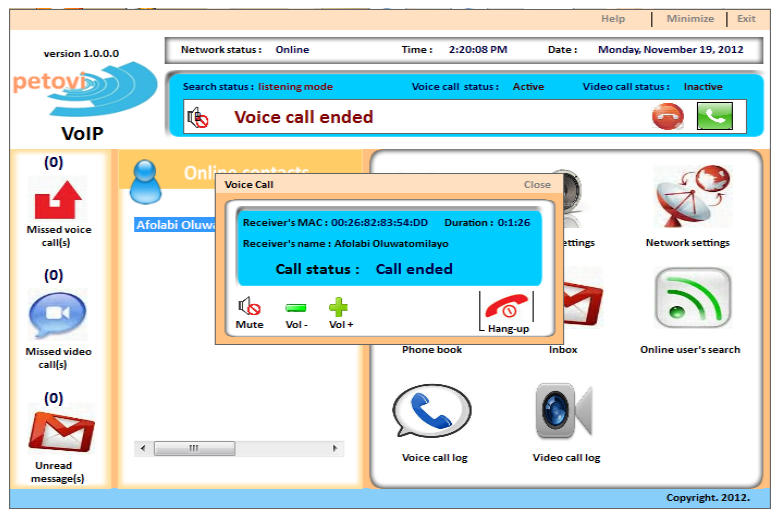

Figure 11: Voice Call Ended after Conversation

\section{Testing of Video Module}

The 'Video Form' was also loaded from the 'Task Selection Bar' by clicking on 'Video Call' option. The 'Video From' was only loaded when the recipient is in the state to receive a call just as the voice module. All operations were similar to that of a voice module except that an attached camera device was activated and there was image streaming alongside voice streaming between the caller and the recipient. Fig.12 and Fig.13 shows a screenshot of the 'Video Form' at the caller's end and at the receiver's end respectively during a video call session.

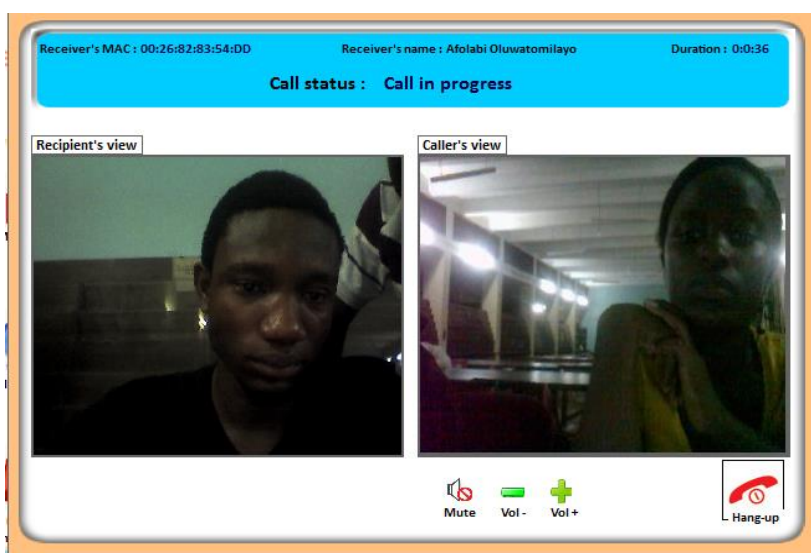

Figure 12: Video Call in Progress (Caller's End)

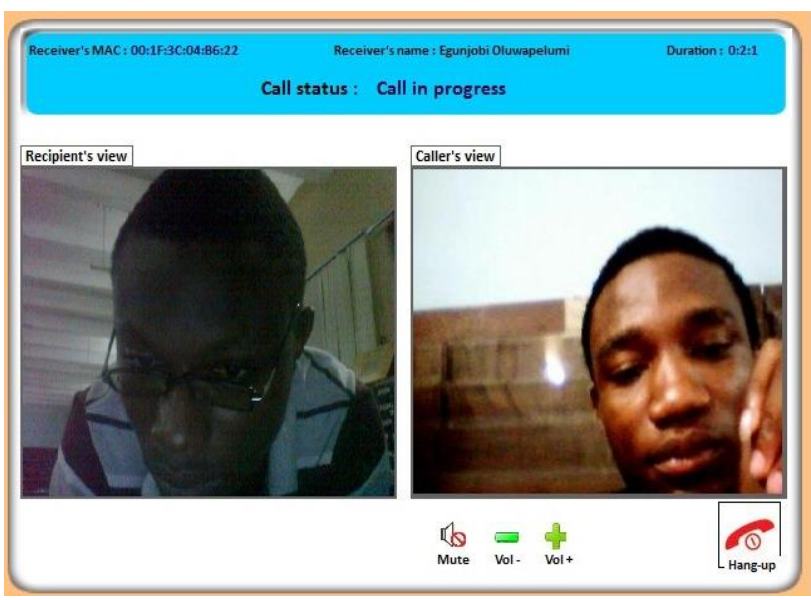

Figure 13: Video Call in Progress (Receiver's End)

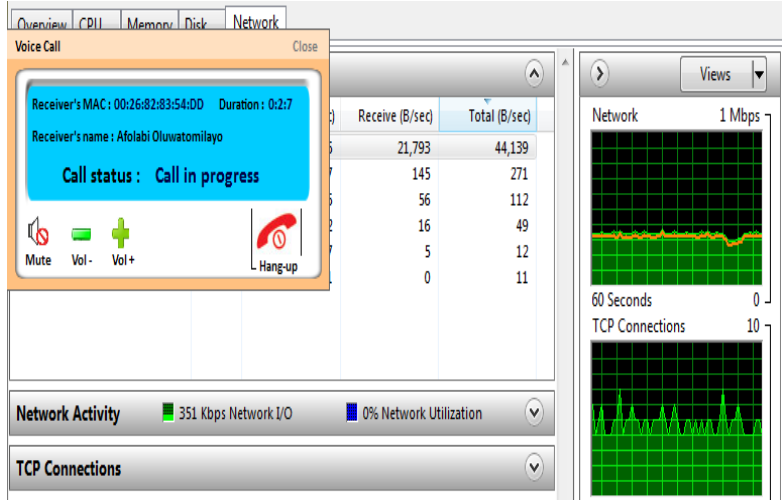

Figure 14: Bandwidth Consumption Statistics during a Call Session

\section{E. WLAN Bandwidth Analysis}

The WLAN bandwidth consumption was observed during a call by two pair session and immediately after the end of the call session. A drop of bandwidth consumption from about $351 \mathrm{Kbps}$ to about $3 \mathrm{Kbps}$ was observed. Fig.14 and 15 show the screenshot of the bandwidth consumption statistics during and immediately after the call session.

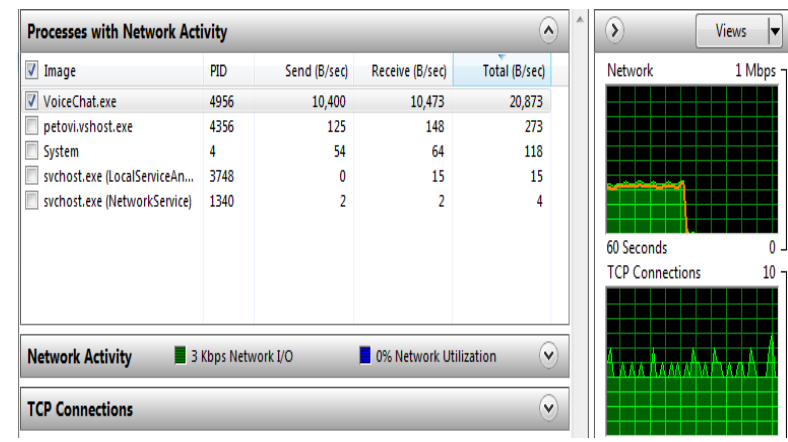

Figure 15: Bandwidth Consumption Statistics after a Call Session

\section{CONCLUSION}

Software-based LAN telephony system is still an emerging field; this work has exposed the advantages it has to offer and how it could be simply implemented in order to reduce the cost spent majorly on intracommunication in any organization. In deploying and testing the system, the establishment, control and termination of a communication sessions such as text messaging, video and voice call was carried out effectively and efficiently with a particular attention paid to the amount of bandwidth usage which has observed did not jeopardize the main functionalities of the existing WLAN backbone.

\section{REFERENCES}

[1] Clif, C. (2002). Bhutan Migration To New Technologies [Wireless VoIP]. BHUTAN: International Telecommunication Union. 
[2] Uys, L. (2009) 'Voice over Internet Protocol (VoIP) as a communication tool in South African Business'. African Journal of Business Management, Vol. 3, No. 3 Pp 89-94.

[3] Ganguly, S. and Bhatnagar, S. (2008). VoIP: Wireless, $P 2 P$ and New Enterprise. New Jersey: Wiley Publication.

[4] Anttalainen, T. (2003). Introduction to Telecommunication Network. Massachusetts: ArtechHouse, Incorporated.

[5] Louis, P. (July, 2004). Voice over Internet Protocol (VoIP): The "Killer" Application? Mind Commerce.

[6] Cox, I (2004). Voice over IP- into the mainstream, [online] white paper, retrieved on 31stOctober 2008 from Juniper Research http://www.juniperresearch.com/shop/products/whit epaper/pdf/VoIP\%20\%20Into\%20\%the\%20Mainstr eam.pdf.

[7] Kolo, G. J.; Dauda, U. S. \& Ali-Nock, G. (2008). Design and Implementation of a Software Intercom on LAN. Minna, Nigeria.

[8] Goode, B. (2002). Voice over Internet Protocol (VoIP), Proceeding of the IEEE, Vol. 90 Pp 1495 1517.

[9] Alvarion. (2006). Implementing VoIP Service Over Wireless Network. Tel Aviv: Alvarion ltd.

[10] Carne, E. B. (2004). A Professional Guide to Data Communication in TCP/IP World. Boston: Artech House Inc.

[11]Clif, C. (2002). Bhutan Migration To New Technologies [Wireless VoIP]. BHUTAN: International Telecommunication Union.

[12] Green, J. H. (2000). Irwin Handbook of Telecommunications. Ohio: McGraw-Hill Professional Publishing.
[13] Hamami, I (2005) Gateway Simulator in a LAN Telephony System, United State Patent and Trademark Office, Sheet 1-5, Patent Number: US 6,898,188 B1.

[14] Xiong H., Guo Y. and Fang Z. (2003) Simple Voice over IP (VoIP) Implementation, ECE Department, University of Florida, Florida.

$[15]$ "Voice over Internet Protocol". Wikipedia [Online]. Retrieved on 2013-08-02 at http://www.en.m.wikipedia.org/wiki/Voice_over_IP.

\section{ACKNOWLEDGEMENTS}

Appreciation goes to STEP-B, University of Ibadan for providing all the necessary equipment for the implementation of the SCP. Also, the implementation was achieved with the help of my project students EgunjobiPelumi, AfolabiTomilayo and Ocheri Victor who dared all the challenges in order to implement the SCP.

\section{AUTHOR PROFILE}

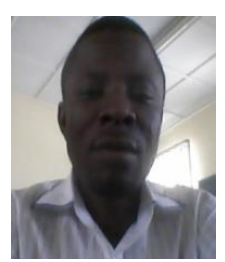

O.O Olakanmireceived theB.Tech in Computer Engineering from LadokeAkintola University of Technology, Ogbomosho 2000 and M.sc in Computer Science from University of Ibadan, Ibadan. He is a lecturer in the Department of Electrical \& Electronic Engineering, University of Ibadan and major in Computer Architecture and Data Security.

How to cite this paper: Olakanmi O.Oladayo,"AWireless Local Area Network Soft Cell Phone (SCP) System with Multimedia and Data Services", IJCNIS, vol.6, no.2, pp.9-15, 2014. DOI: 10.5815/ijcnis.2014.02.02 